\title{
Transmission of tuberculosis to contacts of sputum positive adults in Malawi
}

\author{
Janet M Topley, Dermot Maher, L Nyong'onya Mbewe
}

\begin{abstract}
Over a period of one year from June 1993 to May 1994, 282 children under 6 years old who were household contacts of sputum positive adults with tuberculosis were evaluated in a screening clinic. Of these, $180(63.8 \%)$ had evidence of tuberculosis, a much higher transmission rate than reported elsewhere. HIV seropositivity was $77.4 \%$ in the adult index cases and $18 \%$ in the contact children. No increased infectivity to household contacts was detected in HIV seropositive index adults compared with those who were seronegative. Child tuberculosis contact tracing is essential in these families, where transmission of disease is higher than reported elsewhere, and attention to the health needs of the children may be diminished by the high morbidity and mortality among adult family members.

(Arch Dis Child 1996; 74: 140-143)
\end{abstract}

Keywords: tuberculosis, HIV, contact tracing, tuberculosis transmission.

Since the mid-1980s Malawi has seen a marked increase in annual notification rates for tuberculosis. These have risen from $100 / 100000$ in 1986 to $400 / 100000$ in 1994 (Malawi National Tuberculosis Program, personal communication). Similarly HIV seropositivity has risen from $8 \%$ to $30 \%$ between 1987 and 1994 in urban women tested antenatally in Blantyre. ${ }^{1}$ Children with tuberculosis are usually infected by a sputum positive adult or adolescent in their immediate household, and contact screening is important in detecting early infection in young household members. ${ }^{2}$ Despite this, child contact screening in busy hospitals in developing countries is often neglected because of other priorities. In Malawi, which is one of the poorest countries in Africa with an underfinanced health service, contact tracing was not routine as part of the national tuberculosis programme, which receives support from the International Union against Tuberculosis and Lung Disease in the form of advisors and drug donations.

The strong association between tuberculosis and HIV infection in adults has become clear as the rising prevalence of tuberculosis in subSaharan Africa has closely reflected rising HIV seroprevalence rates. ${ }^{3}$ Adults with tuberculosis/HIV co-infection are thought to be no more infectious than HIV seronegatives since more of them are sputum negative and since sputum sterilisation of the smear positive individuals in response to antituberculosis chemotherapy is reported to be satisfactory. ${ }^{4}$ The transmission of tuberculosis to children in the households of sputum positive adults in Africa has received relatively little recent attention, and the additional aspect of transmissibility from tuberculosis/HIV co-infected adults prompted this study.

\section{Methods}

Appointments were offered to all children under 6 years old in the household of every new tuberculosis sputum positive adult identified at the Queen Elizabeth Central Hospital in Blantyre as part of a new routine contact screening service. On admission of the adult, a child contact screening form was completed listing all child contacts; this was included in the patient's hospital file. All household contacts under 6 years old and any older children with symptoms were offered an appointment for the screening clinic by the district tuberculosis officer (LNM) whose office is on the adult tuberculosis ward. This report concerns only the contacts under the age of 6 years. The screening clinic was run by a consultant paediatrician (JMT) who saw all the patients and read all the Mantoux test results. Mantoux injection, using 1 unit of RT23 Copenhagen solution, was performed by two members of the hospital laboratory staff who were responsible for all Mantoux tests in the hospital. A single PA or AP $x$ ray film of the chest was taken on each child. HIV serology was checked using the Behring ELISA test in as many children as possible, depending on the availability of a parent to provide consent for the test and nursing staff to collect the blood samples. In general, younger and sicker children were more likely to be tested. The study was performed within the limitations of an underfinanced health service with no additional funding; this helps to explain the lack of facilities with which to investigate the children.

Because of the known limitations in both sensitivity and specificity of microscopy and culture of gastric washings, ${ }^{5}$ and local difficulties in performing the test, the diagnosis of tuberculosis was based on consistent symptoms and signs, chest radiology, Mantoux reaction, and response to treatment. Consistent symptoms included cough or fever of more than two weeks duration and failure to thrive, and in children with extrapulmonary tuberculosis the symptoms included abdominal swelling and altered neurological status. Consistent signs included those of collapse/ consolidation or effusion in the chest and the presence of masses or fluid in the abdomen. Central nervous system signs included 
Table 1 Diagnosis of probable tuberculosis

1. Symptoms and signs consistent with the diagnosis and Mantoux $15 \mathrm{~mm}+$.

2. Symptoms and signs consistent with the diagnosis and response to treatment including resolution of symptoms and catch up growth.

3. Abnormal chest radiology and Mantoux $15 \mathrm{~mm}+$.

4. Abnormal chest radiology and response to treatment including resolution of symptoms and catch up growth

meningism and cranial nerve palsies. Chest radiographs were classified as abnormal if there were unequivocal changes consistent with a diagnosis of tuberculosis, such as hilar adenopathy, segmental or lobar collapse-consolidation, or pleural effusion. Mild abnormalities such as perihilar infiltrates were noted but not categorised as abnormal.

\section{CRITERIA FOR THE DIAGNOSIS}

A diagnosis of active clinical tuberculous disease (table 1) was regarded as probable if there were appropriate symptoms and signs or abnormal chest radiology, combined with either (a) a Mantoux reaction of $15 \mathrm{~mm}$ or more, or (b) a demonstrated response to treatment with resolution of symptoms and catch up growth documented on the growth chart.

Catch up growth was defined as an increase of more than 10 centiles on the weight for age chart in response to antituberculosis treatment in outpatients only, since it could be attributed to no other intervention such as nutritional supplementation which might occur in children admitted to hospital.

Tuberculous disease was suspected in children with similar clinical profiles but who failed to fulfil the above criteria because either (a) the Mantoux response was less than $15 \mathrm{~mm}$, or (b) they defaulted from follow up, or (c) because the disease had not yet interfered with growth by the time treatment was begun. ${ }^{6}$

The remaining children were considered unlikely to have tuberculous disease and would have included children with a positive Mantoux response but normal chest radiograph and absence of symptoms or signs of disease.

The Malawi national tuberculosis programme guidelines were followed using rifampicin, isoniazid, and pyrazinamide in the standard ambulatory regimen, and additional streptomycin for severe tuberculosis. Drugs were dispensed by the district tuberculosis officer at monthly intervals and appointments

Table 2 Mantoux results and radiological findings in the 282 children, according to whether tuberculosis was probable, suspected, or unlikely

\begin{tabular}{llcc}
\hline & $\begin{array}{l}\text { No(\%) } \\
\text { probable }\end{array}$ & $\begin{array}{l}\text { No(\%) } \\
\text { suspected }\end{array}$ & $\begin{array}{l}\text { No(\%) } \\
\text { unlikely }\end{array}$ \\
\hline Symptoms reported & $67(71 \cdot 3)$ & $60(69 \cdot 8)$ & $20(19 \cdot 6)$ \\
Cough >2 weeks & $42(44 \cdot 7)$ & $37(43 \cdot 0)$ & $14(13 \cdot 7)$ \\
Failure to thrive & $41(43 \cdot 6)$ & $32(37 \cdot 2)$ & $6(5 \cdot 9)$ \\
Mantoux 0 mm & $27(28 \cdot 7)$ & $39(45 \cdot 3)$ & $81(79 \cdot 4)$ \\
Mantoux 1-9 mm & $13(13 \cdot 8)$ & $21(24 \cdot 4)$ & $21(20 \cdot 6)$ \\
Mantoux 10-14 mm & $13(13 \cdot 8)$ & $26(30 \cdot 2)$ & 0 \\
Mantoux 15+ mm & $41(43 \cdot 6)$ & 0 & 0 \\
Chest $x$ ray abnormal & $48(51 \cdot 1)$ & $45(52 \cdot 3)$ & $6(5 \cdot 9)$ \\
Children with HIV positive source $(\mathrm{n}=105)$ & $33(30 \cdot 6)$ & $39(36 \cdot 1)$ & $33(30 \cdot 6)$ \\
Children with HIV negative source $(\mathrm{n}=37)$ & $13(35 \cdot 1)$ & $10(27 \cdot 0)$ & $14(37 \cdot 8)$ \\
HIV positive children & $10 / 62$ & $13 / 42$ & $2 / 34$ \\
Follow up >1.5 months & $61(64 \cdot 8)$ & $27(31 \cdot 4)$ & $45(44 \cdot 1)$ \\
Total in each diagnostic group & $94(33 \cdot 3)$ & $86(30 \cdot 5)$ & $102(36 \cdot 2)$ \\
\hline
\end{tabular}

for follow up at the clinic were given at one, two, or three monthly intervals depending on the clinical status of the child. Follow up for a period of at least one year was attempted. Statistical analysis was not applied to the data since the diagnostic groups were defined by the variables.

\section{Results}

Seven hundred and seven new sputum positive adults were identified during the period of study and 206 of them arranged for their contacts under 6 years old to attend the clinic. Some of the 707 adults came from the same household so contacts had already been invited, and some had no young household contacts. Appointments were declined for 44 contacts $(13 \cdot 1 \%)$ who lived far from the hospital and had problems with transport, and 10 children (3\%) who were given an appointment failed to attend. In all 282 children from 206 households attended the contact clinic. In $248(88 \%)$, the adult tuberculosis contact was the mother, father, or both parents, indicating the younger adult age group currently affected by tuberculosis in Malawi. Of the 106 adults in whom the HIV serology result was known, $82(77 \cdot 4 \%)$ were seropositive, consistent with results for sputum positive adults at this hospital (AD Harries personal communication).

SOCIOECONOMIC AND NUTRITIONAL STATUS

Eighty seven per cent of the fathers were employed or self employed in the informal sector and the mean number of people sleeping in the child's bedroom was four (range one to 11). The mean percentage expected weight for age (per cent of the median) of the children was $85.9 \%$ in the total group and $99(35.1 \%)$ were underweight (below $80 \%$ of the median). There was no significant difference in nutritional status between children in the three diagnostic categories.

\section{BCG STATUS}

BCG scars were noted in $253(89.7 \%)$ and in only five children $(1.8 \%)$ was it known that no BCG had been given. The remaining 24 children were all reported to have received BCG and this was confirmed from the Under5 -Card in 15; overall 268 (95\%) had documented evidence of BCG immunisation.

\section{SYMPTOMS}

Table 2 shows the numbers in each diagnostic category and the frequency of symptoms in the three categories. The mean duration of symptoms in the probable tuberculosis group was 3.9 months (range $0.25-24$ ) and in the suspected group was 3.8 months (range $0 \cdot 25-20$ ). Failure to thrive may have been a symptom or a sign and in 151 children Under-5-Cards were suitable to assess current growth. In 77 (51\%), growth faltering indicated by failure to gain weight for at least two months was 
documented. Failure to thrive included both documented growth faltering and reported loss of weight.

MANTOUX TESTING AND CHEST RADIOLOGY Table 2 gives the Mantoux results and radiological findings in the three groups. Since most had received BCG, a positive Mantoux reaction was taken as $15 \mathrm{~mm}$ or more, except in HIV seropositive children in whom $10 \mathrm{~mm}$ was taken as positive. Although chest radiology was classified as abnormal in $50 \%$, in only six of the probable group and five of the suspected group were radiographs regarded as entirely normal.

HIV SEROSTATUS

HIV serology was tested in 138 of the children, of whom $25(18 \cdot 1 \%)$ were seropositive. The 138 children who were serotested comprised $66 \%$ of the probable tuberculosis group, $49 \%$ of the suspected tuberculosis group, and $33 \%$ of the unlikely group; the numbers in each group who were seropositive are shown in table 2 . Tuberculous disease was considered probable in 10 of the seropositive children, suspected in 13 , and unlikely in two. Since chronic cough and failure to thrive is a feature of both tuberculosis and AIDS the important findings in these children are the Mantoux reactions and the response to treatment. Four $(16 \%)$ of the 25 HIV seropositive children had Mantoux reactions of 5-9 $\mathrm{mm}$ and another four $(16 \%)$ had reactions of $10 \mathrm{~mm}$ or more. Eight $(32 \%)$ responded well to treatment and all those who responded poorly were below the age of 24 months, whereas five of the eight good responders were older.

INFECTIVITY OF INDEX ADULTS

There were 206 index sputum positive adults and of these $74(35.9 \%)$ had one or more probable child tuberculosis contacts. Sixty eight adults $(33 \%)$ had only tuberculosis unlikely contacts. There was no significant difference in the diagnostic categories of children whose tuberculosis source was HIV seropositive or seronegative (table 2). Of the 36 tuberculous adults with probable child tuberculosis contacts and whose serostatus was known, $27(75 \%)$ were HIV seropositive and nine (25\%) were HIV seronegative. Of the 36 adults with known serostatus and only tuberculosis unlikely child contacts, 28 $(77 \cdot 7 \%)$ were HIV seropositve and eight $(22 \cdot 3 \%)$ were HIV seronegative. There was therefore no evidence of increased transmissibility of tuberculosis by HIV seropositive adults. There was no correlation between the number of individuals sleeping in the same room as the child and risk of transmission, but in the 13 children in whom both parents were currently on antituberculosis treatment only one $(7 \cdot 7 \%)$ was considered unlikely to have tuberculosis.

RESPONSE TO TREATMENT AND FOLLOW UP Table 2 shows the duration of follow up in the three groups. Default from the clinic was high, with only $133(47 \cdot 2 \%)$ attending for follow up beyond six weeks. However examination of their tuberculosis registration cards held in the district tuberculosis office showed that $25 \%$ of those started on antituberculosis treatment who defaulted from the clinic continued to take medication for two months or more after their last clinic visit, and almost half of those who completed treatment defaulted earlier since it was possible to collect drugs from the tuberculosis office on most days without attending the clinic. This meant that adults attending the hospital for another reason could collect antituberculosis drugs for their children at the same time. Six children required admission to hospital for treatment and the remainder were managed as outpatients. The response to treatment was evaluated in 87 children and was good in $64(73.6 \%)$. In six children $(6.9 \%)$ there was no response to treatment, and in the remaining $17(19.5 \%)$ response to treatment was equivocal and could not be used as evidence to support the diagnosis. Weight gain after two months of treatment ranged from $5 \%$ to $50 \%$ of the initial body weight.

\section{Discussion}

The AIDS epidemic in Malawi and elsewhere in Africa ${ }^{7}$ is having serious effects on child morbidity and mortality both from direct consequences of HIV transmission and indirectly from malnutrition and infectious diseases such as tuberculosis. National tuberculosis programmes inevitably concentrate on the public health and cost-effective aspects of the tuberculosis/HIV epidemic, so the needs of children may be neglected since they are generally non-infectious and therefore of no risk to the community. They do, however, represent the potentially infectious adults of the future, and it is clearly more cost effective to treat children before they need admission to hospital. ${ }^{8}$ It is also important to identify those asymptomatic children who will benefit from isoniazid prophylaxis. ${ }^{9}$

Our study indicates a high level of transmission of symptomatic tuberculosis to child contacts, in contrast to earlier studies in Britain and the USA, where no recognisable symptoms were seen in $40-50 \%$ of infected infants and $80-95 \%$ of older children. ${ }^{1011} \mathrm{An}$ Indian study ${ }^{12}$ found a prevalence of active tuberculosis of $20 \%$ in under 5 year old contacts of tuberculosis sputum positive adults in a population which had not received BCG vaccine. A study from Kenya found that $23.8 \%$ of under 6 year old contacts had active tuberculous lesions. ${ }^{13}$ The higher transmission rate in this report may be a result of worse socioeconomic conditions associated with domestic overcrowding and poor nutrition, a greater degree of infectivity in the index cases, or increased genetic susceptibility to tuberculosis. It is possible that the children who attended this clinic were selected because they had symptoms, but we have no evidence for this and almost $50 \%$ who attended were asymptomatic. A possible selection bias is that index 
patients may have missed the contact procedure at times when the ward was busy often with three adult patients allocated to each bed - but this would affect both infected and non-infected contacts similarly. The use of only 1 tuberculin unit, which because of local strictures was out of our control, may have resulted in underdiagnosis of tuberculous infection, since the use of 2 or 5 . units would almost certainly have produced more positive Mantoux reactions. We had evidence that tuberculous disease was underdiagnosed initially, in that three children who had no symptoms or signs and normal radiology showed growth acceleration when started on isoniazid prophylaxis, suggesting the presence of active disease.

It is reassuring to find no evidence of increased transmissibility of tuberculosis by HIV seropositive adults, consistent with other reports on their usually normal response to antituberculosis chemotherapy. ${ }^{3}$ It is surprising to find no clear correlation with domestic overcrowding but this may be explained by the fact that most of the children slept in the same room as the index patient, which is more likely to affect the degree of exposure of the child to infection. It is noteworthy that only two $(8 \%)$ of the 25 seropositive children were thought unlikely to have tuberculosis, suggesting that, once exposed, the HIV positive child is likely to develop active tuberculosis.

The children in this study are particularly at risk because at least two thirds will lose one or both parents as a result of AIDS, and meanwhile they are subject to the problems of chronic illness and economic deprivation within the family. Eighty per cent of the children were HIV seronegative, so should recover from tuberculosis given appropriate care. The long duration of symptoms in these children attests to the diminished attention to their needs and the importance of case finding in this vulnerable age group.

Our findings suggest that in spite of high BCG coverage the young contacts of sputum positive adults are at considerable risk of developing tuberculous disease. Since only six of 180 children with probable or suspected tuberculosis required admission to hospital it is clear that identifying them earlier by contact tracing allowed cheaper ambulatory care. Those children who showed no evidence of disease were offered six months of isoniazid prophylaxis after the first year of this study, when results indicated the need for this, particularly in view of the delayed development of tuberculous disease while under the clinic observation. Our findings were reported locally and regionally, with the recommendation that all under 6 year old contacts and any older children with symptoms should be examined clinically and radiologically, and that isoniazid prophylaxis should be offered to all those without disease in the younger group and to all Mantoux positive older children without evidence of disease.

Our thanks to Dr David Brewster and Professor A D Harries for their helpful comments on the manuscript.

1 Taha TE, Canner JK, Wangel A-M, et al. Research on human immunodeficiency virus (HIV) in Malawi: the Johns Hopkins University - Ministry of Health (JHUMOH) Project. Malawi Med f 1994; 10: 6-11.

2 British Thoracic and Tuberculosis Association. A study of standardized contact procedure in tuberculosis. Tubercle 1978; 59: 245-59.

3 Narain JP, Raviglione MC, Kochi A. HIV-associated tuberculosis in developing countries: epidemiology and strategies for prevention. Tubercle Lung Dis 1992; 73: 311-21.

4 Baende E, Klausner J, Lelo U, et al. Characterisation of transmitters of $\mathbf{M}$ tuberculosis (M.TB) in Zaire by HIV serostatus, level of immunosuppression and clinica status. VII International Conference on AIDS, Florence, Italy 16-20 June 1991, abstract MC3253:307.

5 Kibel MA, Hussey G. Problems in the diagnosis of childhood tuberculosis. S Afr Med F 1990; 77: 379-80.

6 World Health Organisation. Provisional guidelines for the diagnosis and classification of EPI Target diseases for primary
health care surveillance and special studies. (EPI/GEN/83/4.) health care surveillance and

7 Chintu C, Bhat G, Luo C, et al. Seroprevalence of human immunodeficiency virus type I infection in Zambian children with tuberculosis. Pediatr Inf Dis $\mathcal{F}$ 1993; 12: 499-504.

8 Starke JR. Modern approach of the diagnosis and treatment of tuberculosis in children. Pediatr Clin North Am 1988, 35: 441-64

9 Mount FW, Ferebee SH. Preventive effects of isoniazid in the treatment of primary tuberculosis in children. $N$ Engl f Med 1961; 265: 713-21.

10 Payne M. In: Miller FJW, Seale RME, Taylor MD, eds. Tuberculosis in children. Boston: Little, Brown, 1963.

11 Cammock RM, Miller FJW. Tuberculosis in young children. Lancet 1953; i: 158-60.

12 Devadatta S, Dawson JY, Fox W, et al. Attack rate of tuberculosis in a five year period among close family contacts of culosis in a five year period among close family contacts of tuberculous patients under domiciliary treatment with
isoniazid plus PAS or isoniazid alone. Bull WHO 1970; 42: $337-51$.

13 WHO Tuberculosis Chemotherapy Centre, Nairobi. An investigation of household contacts of open cases of pulmonary tuberculosis amongst the Kikuyu in Kiambu, Kenya. Bull World Health Organ 1961; 25: 831-50. 